\title{
Chapter 3 \\ 3D Multiscale Modelling of Angiogenesis and Vascular Tumour Growth
}

\author{
H. Perfahl, H.M. Byrne, T. Chen, V. Estrella, T. Alarcón, A. Lapin, \\ R.A. Gatenby, R.J. Gillies, M.C. Lloyd, P.K. Maini, M. Reuss, \\ and M.R. Owen
}

\begin{abstract}
We present a three-dimensional, multiscale model of vascular tumour growth, which couples nutrient/growth factor transport, blood flow, angiogenesis, vascular remodelling, movement of and interactions between normal and tumour cells, and nutrient-dependent cell cycle dynamics within each cell. We present computational simulations which show how a vascular network may evolve and
\end{abstract}

The chapter is based on Perfahl et al., 2011, Multiscale Modelling of Vascular Tumour Growth in 3D: The Roles of Domain Size and Boundary Conditions. PLoS ONE 6(4): e14790. doi:10.1371/ journal.pone.0014790

H. Perfahl $(\bowtie) \cdot$ A. Lapin • M. Reuss

Center for Systems-Biology, University of Stuttgart, Stuttgart, Germany

e-mail: perfahl@ibvt.uni-stuttgart.de; lapin@ibvt.uni-stuttgart.de; reuss@ibvt.uni-stuttgart.de

H.M. Byrne

Oxford Centre for Collaborative Applied Mathematics, Department of Computer Science,

University of Oxford, Oxford, UK

e-mail: helen.byrne@maths.ox.ac.uk

T. Chen • V. Estrella • R.A. Gatenby • R.J. Gillies • M.C. Lloyd

H. Lee Moffitt Cancer Center \& Research Institute, Tampa FL 33612, USA

e-mail: tingan.chen@moffitt.org; veronica.estrella@moffitt.org; robert.gatenby@moffitt.org;

robert.gillies@moffitt.org; mark.lloyd@moffitt.org

T. Alarcón

Centre de Recerca Matemàtica, Campus de Bellaterra, Barcelona, Spain

e-mail: talarcon@crm.cat

P.K. Maini

Centre for Mathematical Biology, Mathematical Institute and Oxford Centre for Integrative Systems Biology, Department of Biochemistry, University of Oxford, Oxford, UK

e-mail: maini@maths.ox.ac.uk

M.R. Owen

Centre for Mathematical Medicine and Biology, School of Mathematical Sciences,

University of Nottingham, Nottingham, UK

e-mail: markus.owen@nottingham.ac.uk 
interact with tumour and healthy cells. We also demonstrate how our model may be combined with experimental data, to predict the spatio-temporal evolution of a vascular tumour.

\subsection{Introduction}

Angiogenesis marks an important turning point in the growth of solid tumours. Avascular tumours rely on diffusive transport to supply them with the nutrients they need to grow, and, as a result, they typically grow to a maximal size of several millimetres in diameter. Growth stops when there is a balance between the rate at which nutrient-starved cells in the tumour centre die and the rate at which nutrient-rich cells on the tumour periphery proliferate. Under low oxygen, tumour cells secrete angiogenic growth factors that stimulate the surrounding vasculature to produce new capillary sprouts that migrate towards the tumour, forming new vessels that increase the supply of nutrients to the tissue and enable the tumour to continue growing and to invade adjacent healthy tissue. At a later stage, small clusters of tumour cells may enter the vasculature and be transported to remote locations in the body, where they may establish secondary tumours and metastases [12].

In more detail, the process of angiogenesis involves degradation of the extracellular matrix, endothelial cell migration and proliferation, capillary sprout anastomosis, vessel maturation and adaptation of the vascular network in response to blood flow [29]. Angiogenesis is initiated when hypoxic cells secrete tumour angiogenic factors (TAFs), such as vascular endothelial growth factor (VEGF) $[27,13]$. The TAFs are transported through the tissue by diffusion and stimulate the existing vasculature to form new sprouts. The sprouts migrate through the tissue, responding to spatial gradients in the TAFs by chemotaxis. When sprouts connect to other sprouts or to the existing vascular network via anastomosis, new vessels are created. Angiogenesis persists until the tissue segment is adequately vascularised. The diameter of perfused vessels changes in response to a number of biomechanical stimuli such as wall shear stress (WSS) and signalling cues such as VEGF $[31,26]$. For example, vessels which do not sustain sufficient blood flow will regress and be pruned from the network $[10,28]$.

Tumour growth and angiogenesis can be modelled using a variety of approaches (for reviews, see [18, 35]). Spatially averaged models can be formulated as systems of ordinary differential equations (ODEs) (see [8, 7]). Alternatively, a multiphase approach can be used to develop a spatially structured continuum model that describes interactions between tumour growth and angiogenesis and is formulated as a mixed system of partial differential equations (PDEs) [9]. Alternatively, a 2D stochastic model that tracks the movement of individual endothelial cells to regions of high VEGF concentration is introduced in [6]. Following [6], McDougall and co-workers [19] have developed a model for angiogenesis and vascular adaptation in which the tissue composition is static and 
attention focusses on changes in the vasculature. This framework has been extended by Stéphanou et al. [33] to produce 3D simulations of angiogenesis and vascular adaptation. More recently, Macklin et al. [17] coupled a multiphase model to a discrete model of angiogenesis that accounts for blood flow, nonNewtonian effects and vascular remodelling. The models are coupled in two ways: via hydrostatic pressure which is generated by the growing tumour and acts on the vessels and via oxygen which is supplied by the vessels and stimulates growth. Lloyd et al. [16] have developed a model for neoplastic tissue growth which accounts for blood and oxygen transport and angiogenic sprouting. The strain (local deformation) in the tumour tissue is assumed to be an increasing function of the local oxygen concentration. In separate work, Owen et al. [20], building on the work of Alarcón and co-workers [1, 2, 3, 4], proposed a 2D multiscale model for vascular tumour growth which combines blood flow, angiogenesis, vascular remodelling and tissue scale dynamics of multiple cell populations as well as the subcellular dynamics (including the cell cycle) of individual cells. More recently, this framework was extended by Owen et al. [21] to analyse synergistic anti-tumour effects of combining a macrophage-based, hypoxia-targeted, gene therapy with chemotherapy.

While several two-dimensional models of angiogenesis consider tumour growth, few groups account for vascular tumour growth in three spacedimensions. In an extension to work by Zheng at al. [37], Frieboes et al. [14] couple a mixture model to a lattice-free continuous-discrete model of angiogenesis [24] to study vascular tumour growth. However, the effects of blood flow and vascular remodelling are neglected. Lee et al. [15] studied tumour growth and angiogenesis, restricting vessel sprouting to the tumour periphery and surrounding healthy tissue. They incorporated vessel dilation and collapse in the tumour centre and analysed the micro-vessel density within the tumour. Building on work by Schaller and Meyer-Hermann [30], Drasdo et al. [11] developed a lattice-free model for 3D tumour growth and angiogenesis that includes biomechanically induced contact inhibition and nutrient limitation. However, they do not consider an explicit cell cycle model, they neglect the effects of flow-induced vascular remodelling and they ignore interactions between normal and tumour cells. Similarly, Shirinifard et al. [32] present a 3D cellular Potts model of tumour growth and angiogenesis in which blood flow and vascular remodelling are neglected, as are the cell cycle and competition between normal and tumour cells.

In this chapter, we present a 3D multiscale model of angiogenesis and vascular tumour growth, based on Owen et al. [20]. In Sect. 3.2, the mathematical model and the associated computational algorithm are introduced. Computational simulations are presented in Sect. 3.3. There we start by illustrating the growth of a tumour, nested in healthy tissue with two straight initial vessels. We also show how vascular networks derived from experimental data can be integrated in our model. 


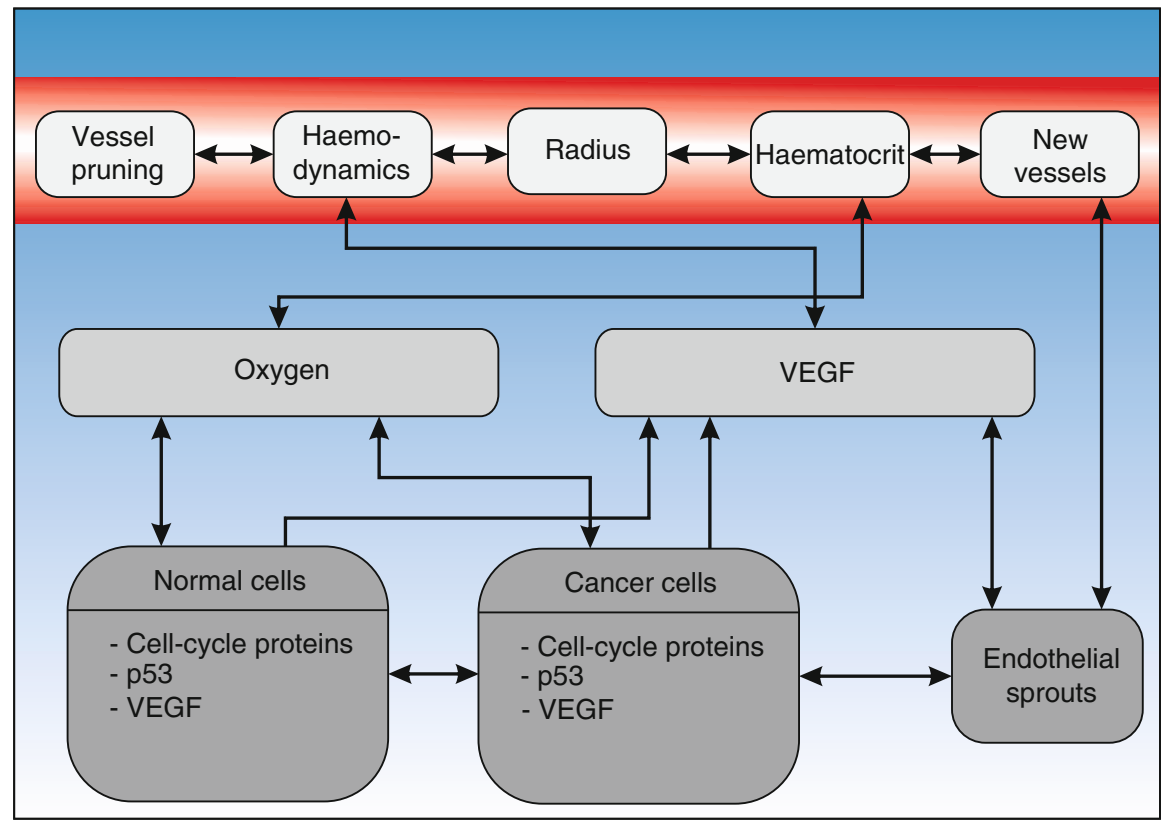

Fig. 3.1 Multiscale model overview (interaction diagram). This figure shows the connections between the different modelling layers. In the subcellular layer, the cell cycle protein concentrations and the p53 and VEGF concentrations are modelled via systems of coupled ordinary differential equations. The local external oxygen concentration influences the duration of the cell cycles. Cells consume oxygen, and produce VEGF in the case of hypoxia. Extracellular VEGF also influences the emergence of endothelial sprouts and their biased random walk towards hypoxic regions. If endothelial sprouts connect to other sprouts or the existing vascular network, new vessels form. Vessel diameter is influenced by the local oxygen concentration and flowrelated parameters, such as pressure and wall shear stress. The vascular network delivers oxygen throughout the tissue

\subsection{Multiscale Model}

The computational model that we use describes the spatio-temporal dynamics of a tumour located in a vascular host tissue. Cells are represented as individual entities (agent-based approach), each with their own cell cycle and subcellular-signalling machinery. Nutrients are supplied by a dynamic vascular network, which is subject to remodelling and angiogenesis. Interactions between the different layers are depicted in Fig. 3.1.

Our model is formulated on a regular grid that subdivides the simulation domain into lattice sites. Each lattice site can be occupied by several biological cells whose movement on the lattice is governed by reinforced random walks, and whose proliferation is controlled by a subcellular cell cycle model. The vascular network consists of vessel segments connecting adjacent nodes on the lattice, with defined 
inflow and outflow nodes with prescribed pressures. We also specify the amount of haematocrit entering the system through the inlets. The vessel network evolves via (1) sprouting of tip cells with a probability that increases with the local VEGF concentration, (2) tip cell movement described by a reinforced random walk, and (3) new connections formed via anastomosis. In addition, vessel segments with low WSS for a certain time are pruned away. Elliptic reaction-diffusion equations for the distributions of oxygen and VEGF are implemented on the same spatial lattice using finite difference approximations, and include source and sink terms based on the location of vessels (which act as sources of oxygen and sinks of VEGF) and the different cell types (e.g. cells act as sinks for oxygen and hypoxic cells as sources of VEGF).

In summary, after initialising the system, the diffusible fields, cellular and subcellular states are updated (including cell division and movement), before the vessel network is updated; this process is then repeated until the simulation ends.

A more detailed description of the mathematical model is presented in the following subsections. We start at the smallest spatial scale, namely, the subcellular layer. Then the cellular and diffusible layers are introduced, before the vascular layer. Interactions between these layers are highlighted in the final part of this section where the computational algorithm is presented. The parameter values can be found in Perfahl et al. [22].

\subsubsection{Subcellular Layer}

Coupled systems of non-linear ODEs are used to model progress through the cell cycle, and changes in expression levels of p53 and VEGF [3]. In practice, the cell cycle can be divided into four phases: during $G_{1}$, the cell is not committed to replication, but if conditions are favourable, it may enter the $S$ (synthesis) phase, in which DNA replication takes place. During the $G_{2}$ phase, further growth, and DNA and chromatid alignment occur, before the cell divides during $M$ (mitosis) phase. For the cell cycle, we consider the cell mass $M$ and the proteins cycCDK (cyclinCDK complex), Cdh1 (Cdh1-APC complex), p27 and npRB (non-phoshorylated retinoblastoma protein). The cell cycle model that we use focuses on the $G_{1}-S$ transition. It extends an earlier model due to Tyson and Novak [36] by accounting for the p27-mediated effect that hypoxia has on the cell cycle [2]. Using square brackets to represent intracellular protein concentrations, we have

$$
\begin{gathered}
\frac{\mathrm{d}[\mathrm{Cdh} 1]}{\mathrm{d} t}=\frac{\left(1+b_{3}[\mathrm{npRB}]\right)(1-[\mathrm{Cdh} 1])}{J_{3}+1-[\mathrm{Cdh} 1]}-\frac{b_{4} M[\mathrm{cycCDK}][\mathrm{Cdh} 1]}{J_{4}+[\mathrm{Cdh} 1]}, \\
\frac{\mathrm{d}[\mathrm{cycCDK}]}{\mathrm{d} t}=a_{4}-\left(a_{1}+a_{2}[\mathrm{Cdh} 1]+a_{3}[\mathrm{p} 27]\right)[\mathrm{cycCDK}],
\end{gathered}
$$




$$
\begin{gathered}
\frac{\mathrm{d} M}{\mathrm{~d} t}=\eta M\left(1-\frac{M}{M^{*}}\right), \\
\frac{\mathrm{d}[\mathrm{p} 27]}{\mathrm{d} t}=c_{1}\left(1-\chi \frac{M}{M^{*}}\right)-\frac{c_{2} c_{02}}{B+c_{02}}[\mathrm{p} 27], \\
\frac{\mathrm{d}[\mathrm{npRB}]}{\mathrm{d} t}=d_{2}-\left(d_{2}+d_{1}[\mathrm{cycCDK}]\right)[\mathrm{npRB}] ;
\end{gathered}
$$

where $b_{3}, J_{3}, b_{4}, J_{4}, a_{1}, a_{2}, a_{3}, a_{4}, \eta, M^{*}, c_{1}, c_{2}, B, \chi, d_{1}$ and $d_{2}$ are constants, specified in [22].

In (3.1)-(3.5), when $M$ is small, the cell is maintained in a state corresponding to $G_{1}$ for which levels of Cdh1 are high and levels of cycCDK are low. Growth in the cell mass increases $\mathrm{Cdh} 1$ degradation and reduces $\mathrm{p} 27$ production, so that cycCDK increases. This leads to inhibition of $\mathrm{npRB}$ and $\mathrm{Cdh} 1$ and, hence, positive feedback on cycCDK. At a certain point, corresponding to the $G_{1}-S$ transition, the state with high Cdh1 and low CDK is lost, and a state with low Cdh1 and high cycCDK is attained. Finally, when Cdh1 levels are sufficiently low and CDK levels sufficiently high, cell division occurs. The external $\mathrm{O}_{2}$ concentration $c_{02}$ couples the subcellular and diffusible scales by influencing progress through the cell cycle. Decreasing $c_{02}$ reduces p27 degradation, and the resulting increase in levels of p27 counteracts the effect of the increasing mass on cycCDK. In particular, if $c_{02}$ levels are sufficiently low, the $G_{1}-S$ transition cannot occur. Further details about the model can be found in $[2,3]$.

The intracellular concentration of p53 regulates normal cell apoptosis, and that of VEGF controls VEGF release by normal cells. The dynamics of p53 and VEGF are coupled to one another and to the extracellular oxygen concentration, as described by the following differential equations:

$$
\begin{gathered}
\frac{\mathrm{d}[\mathrm{p} 53]}{\mathrm{d} t}=k_{7}-k_{7}^{\prime} \frac{c_{02}}{K_{\mathrm{p} 53}+c_{02}}[\mathrm{p} 53], \\
\frac{\mathrm{d}[\mathrm{VEGF}]}{\mathrm{d} t}=k_{8}+k_{8}^{\prime \prime} \frac{[\mathrm{p} 53][\mathrm{VEGF}]}{J_{5}+[\mathrm{VEGF}]}-k_{8}^{\prime} \frac{c_{02}}{K_{\mathrm{VEGF}}+c_{02}}[\mathrm{VEGF}]
\end{gathered}
$$

with the constants $k_{7}, k^{\prime}{ }_{7}, K_{\mathrm{p} 53}, k_{8}, k_{8}^{\prime}, k_{8}^{\prime}, J_{5}$ and $K_{\mathrm{VEGF}}$ (see [22]). The ODEs (3.1)-(3.7) are solved subject to the initial conditions specified in [22], using the open source CVODE library. ${ }^{1}$

\footnotetext{
${ }^{1}$ https://computation.llnl.gov/casc/sundials/main.html.
} 
Cell death, quiescence and proliferation are determined by a cell's internal protein concentrations. We apply the following rules to identify the different cell states and show their application for a particular cell $i$ (intracellular concentrations of the cell $i$ are denoted by [ $\cdot](i))$. In normal cells, cell death occurs if [p53] (i) $>\mathrm{p} 53_{\mathrm{THR}}(i)$, where $\mathrm{p} 53_{\mathrm{THR}}(i)$ is the maximal threshold they can sustain before undergoing apoptosis, and is given by

$$
\mathrm{p} 53_{\mathrm{THR}}(i)=\left\{\begin{array}{l}
\mathrm{p} 53_{\mathrm{THR}}^{\text {high }} \text { for } \rho_{\text {normal }}(i)>\rho_{\mathrm{THR}} \\
\mathrm{p} 53_{\mathrm{THR}}^{\text {low }} \text { for } \rho_{\text {normal }}(i) \leq \rho_{\mathrm{THR}}
\end{array} .\right.
$$

We define the set of cells in the neighbourhood of cell $i$ as $\Theta_{i}$. The normal cell ratio in (3.8) is given by

$$
\rho_{\text {normal }}(i)=\frac{\sum_{k \in \Theta_{i}} \text { normal cells at site } k}{\sum_{k \in \Theta_{i}} \text { normal or cancer cells at site } k} .
$$

Definition (3.9) accounts for the fact that healthy cells are more likely to die if they live in a tumour environment. This can be caused by the altered microenvironment in tumours. Tumour cells enter quiescence if [p27] $(i)>\mathrm{p} 27_{e}$ or leave quiescence if [p27] $(i)<\mathrm{p} 27_{l}$. If a cancer cell is in quiescence for too long $\left(>T_{\text {death }}\right)$, the cell dies. It should be noted that cancer cell death is not influenced by p53.

The condition to be satisfied for the proliferation of cells is

$$
[\mathrm{Cdh} 1](i)<\mathrm{Cdh}_{\mathrm{THR}} \text { and }[\mathrm{cycCDK}](i)>\operatorname{cycCDK}_{\mathrm{THR}} \text {. }
$$

The daughter cell is placed in the current location if there is free space; otherwise, it is moved to an empty neighbour location with a high oxygen concentration. If there is no free space in the neighbour CA-cells, the parent cell dies and no daughter is produced.

\subsubsection{Cellular Layer}

The following section focuses on the creation and movement of new vessels. For a detailed description, see Owen et al. [20]. New sprouts form at site $i$ (which must be a vessel site) with probability $\mathscr{P}_{i}^{\text {sprout }}$ where

$$
\mathscr{P}_{i}^{\text {sprout }}=\frac{P_{\text {max }}^{\text {sprout }} c_{\text {VEGF }}}{V_{\text {sprout }}+c_{\mathrm{VEGF}}} \Delta t,
$$

with the timestep size $\Delta t$. Since VEGF stimulates sprout formation, the probability of sprouting is assumed to increase with the VEGF concentration, $c_{\text {VEGF. }}$. The maximum sprouting probability is $P_{\max }{ }^{\text {sprout }}$, and $V_{\text {sprout }}$ is a constant. New sprouts 
can only emerge if sufficient space is available. Around the base of each sprout, a radius of exclusion is defined, in which new sprouts cannot occur. For the vessel tip cells, we define $\mathscr{P}(i \rightarrow j)$ as the probability of moving from $i$ to $j$, to be

$$
\mathscr{P}(i \rightarrow j)=\frac{\Delta t D}{d_{i j}^{2} \Delta x^{2}} \frac{\left(N_{\mathrm{m}}-N_{j}\right)\left(1+\gamma \frac{c_{\mathrm{VEGF}, j}-c_{\mathrm{VEGF}, i}}{d_{i j} \Delta x}\right)}{\sum_{k \in \Omega_{i}}\left(N_{\mathrm{m}}-N_{k}\right)+N_{m}-N_{i}+N_{\mathrm{m}} M_{\mathrm{c}}}
$$

for $i \neq j \in \Omega_{i}$. Herein, $D$ represents the cell motility; $\Delta x$ the CA-lattice size; $N_{\mathrm{m}}$ is the maximal carrying capacity of the cell type attempting to move; $N_{i}$ is the number of cells; $M_{\mathrm{c}}$ is a constant and $c_{\mathrm{VEGF}, i}$ the VEGF level at site $i \gamma$ is the chemotactic sensitivity and $\Omega_{i}$ is the set of sites in the neighbourhood of $i$, not including $i$ itself. The probabilities are weighted with the distance between lattice site $i$ and $j$, written as $d_{i j}$. In the three-dimensional case, $\Omega_{i}$ has at most 26 neighbour elements for each lattice point $i$. We set the probability to zero if an endothelial cell crosses a vessel. The probability of not moving is

$$
\mathscr{P}(i \rightarrow i)=1-\sum_{\substack{j, k \in \Omega_{i} \\ j \neq k}} \mathscr{P}(j \rightarrow k) .
$$

Whenever a tip cell moves to another location, an endothelial cell remains at the former lattice site. This is equivalent to the snail-trail concept also applied in $[5,34,23]$. A sprout dies if it does not connect to another sprout or the existing vasculature within a certain time period.

\subsubsection{Diffusible Layer}

The diffusible layer facilitates the coupling between the vascular and subcellular layers. We consider two diffusible components in our model, namely, oxygen and VEGF, and denote their concentrations by $c_{\mathrm{VEGF}}$ and $c_{02}$, respectively. The vascular system acts as an oxygen source, while the normal and tumour cells act as sinks. This behaviour is described by the following, quasi-stationary, reaction-diffusion equation:

$$
D_{02} \nabla^{2} c_{02}+2 \pi \tilde{R}(t, \mathbf{x}) P_{02}\left(c_{02}^{\text {blood }}-c_{02}\right)-k_{02}(t, \mathbf{x}) c_{02}=0
$$

with the diffusion coefficient of oxygen, $D_{02}$. In (3.14), the vessel radius indicator function $\tilde{R}$ returns the vessel radius if a vessel is present at position $\mathbf{x}$ and zero otherwise. Equation (3.14) also accounts for the vessel permeability to oxygen $\left(P_{02}\right)$, the blood oxygen concentration $c_{02}{ }^{\text {blood }}$ and the cell-type-dependent oxygen consumption rate $k_{02}$. If cells become hypoxic or quiescent, they start to secrete 
VEGF, which then can be removed by the vasculature. The concentration of VEGF is determined by

$$
D_{\mathrm{VEGF}} \nabla^{2} c_{\mathrm{VEGF}}-2 \pi \tilde{R}(t, \mathbf{x}) P_{\mathrm{VEGF}} c_{\mathrm{VEGF}}+k_{\mathrm{VEGF}}(t, \mathbf{x})-\delta_{\mathrm{VEGF}} c_{\mathrm{VEGF}}=0,
$$

wherein $D_{\mathrm{VEGF}}$ is the diffusion coefficient of VEGF, $P_{\mathrm{VEGF}}$ the permeability of the vessels to VEGF, $k_{\mathrm{VEGF}}$ the cell-type-dependent VEGF production rate and the

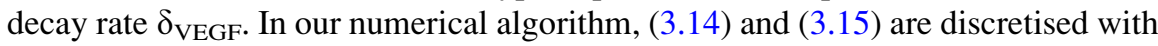
a finite difference scheme, and the resulting sparse linear system of equations is solved with a GMRES-solver.

In case of a non-periodic simulation domain, it is assumed that there is no flux of diffusible substances over the boundary, and thus, homogeneous Neumann boundary conditions are imposed. For simulations in a periodic domain, we apply periodic boundary conditions for the calculation of diffusible substance concentrations.

\subsubsection{Vascular Layer}

We follow very closely the work of Secomb et al. and refer the reader to [26] for full details. We assume a laminar Poiseuille flow in each vessel. The flux $\dot{Q}_{i}$ through vessel $i$ is given by

$$
\dot{Q}_{i}=\frac{\pi R_{i}^{4}}{8 \mu\left(R_{i}, H_{i}\right) L_{i}} \Delta P_{i},
$$

where $\Delta P_{i}$ is the pressure difference at the vessel segment $i, L_{i}$ the vessel length, $\mu\left(R_{i}, H_{i}\right)$ is the radius $R_{i}$ and haematocrit $H_{i}$ dependent blood viscosity [26]. In (3.16), we can identify the resistance of vessel $i$ by $\operatorname{Res}_{i}=8 \mu\left(R_{i}, H_{i}\right) L_{i} /\left(\pi R_{i}^{4}\right)$. In (3.16), the blood viscosity is defined by

$$
\mu(R, H)=\mu_{0} \mu_{\mathrm{rel}}(R, H)
$$

where $\mu_{0}$ is a positive constant,

$$
\begin{gathered}
\mu_{\text {rel }}(R, H)=\left[1+\left(\mu_{0.45}(R)-1\right) \frac{(1-H)^{C}-1}{(1-0.45)^{C}-1}\left(\frac{2 R}{2 R-1.1}\right)^{2}\right]\left(\frac{2 R}{2 R-1.1}\right)^{2} \\
\mu_{0.45}(R)=6 \mathrm{e}^{-0.17 R}+3.2-2.44 \mathrm{e}^{-0.06(2 R)^{0.645}}
\end{gathered}
$$

and 


$$
C=C(R)=\left(0.8+\mathrm{e}^{-0.15 R}\right)\left(-1+\frac{1}{1+10^{-11}(2 R)^{12}}\right)+\frac{1}{1+10^{-11}(2 R)^{12}} .
$$

Using (3.16)-(3.20), we can calculate the flux through each vessel segment in terms of the pressure at each junction of the vascular tree. At any node of the vascular network, the total flow into that node must balance the total flow out of that node. With the pressures at each inlet and outlet ( $P_{\text {in }}$ and $P_{\text {out }}$, respectively) prescribed, we obtain a linear system of equations for the pressures at each vessel node. This system is solved with the direct SuperLU solver. ${ }^{2}$

When updating the vascular network, there are two different timescales of interest, the timescale for flow and the timescale for vascular adaptation. While changes in flow may be rapid, we assume that vascular adaptation occurs on the same timescale as endothelial cell movement and cell division. Consequently, we model the temporal evolution of a vessel segment's radius by applying the following discretised ODEs

$$
R(t+\Delta t)=R(t)+\alpha_{R} \Delta t R(t)\left(S_{\mathrm{h}}+S_{\mathrm{m}}-k_{\mathrm{s}}\right),
$$

where $\Delta t$ is the timestep size and the updated radius must satisfy the constraint $R_{\min } \leq R(t+\Delta t) \leq R_{\max }$. The factor $\alpha_{R}$ that appears in (3.21) relates the stimuli to our timestep size $\Delta t$. In the absence of any details on the rate of vascular adaptation (since all previous studies of which we are aware consider quasi-steady state vessel radii), we set $\alpha_{R}=3.3 \times 10^{-6} \mathrm{~min}^{-1}$ so that the rate of change of the vessel radius is typically less than $10 \%$ per hour. $k_{\mathrm{s}}$ is the shrinking tendency of a vessel which takes into account that vessels tend to regress in the absence of stimuli. $S_{\mathrm{h}}$ and $S_{\mathrm{m}}$ are haemodynamic and metabolic stimuli for vascular adaptation:

\section{- Haemodynamic stimulus:}

$$
S_{\mathrm{h}}=\log \left(\tau_{\mathrm{w}}+\tau_{\mathrm{ref}}\right)-k_{p} \log ((\tau(P)),
$$

with the WSS

$$
\tau_{\mathrm{w}}=\frac{R \Delta P}{L}
$$

the constant reference WSS $\tau_{\text {ref }}$ and the corresponding set point pressure of the WSS $\tau(P)$, described by the empirical function

$$
\tau(P)=100-86 \exp \left(-5000[\log (\log P)]^{5.4}\right)
$$

\footnotetext{
${ }^{2}$ http://crd.lbl.gov/ xiaoye/SuperLU/.
} 
- Metabolic stimulus:

$$
S_{\mathrm{m}}=k_{m}\left(c_{\mathrm{VEGF}}\right) \log \left(\frac{\dot{Q}_{\mathrm{ref}}}{\dot{Q} H+a \dot{Q}_{\mathrm{ref}}}+1\right)
$$

with

$$
k_{m}\left(c_{\mathrm{VEGF}}\right)=k_{m}^{0}\left(1+k_{m}^{\mathrm{VEGF}} \frac{c_{\mathrm{VEGF}}}{V_{0}+c_{\mathrm{VEGF}}}\right)
$$

where $\dot{Q}_{\text {ref }}, a, k_{m}{ }^{0}, k_{m}{ }^{\text {VEGF }}$ and $V_{0}$ are parameters. $\dot{Q}_{\text {ref }}$ is a reference flow, and the term $a \dot{Q}_{\text {ref }}$ (where $a$ is a small parameter) in the denominator of (3.25) is introduced to avoid extreme vessel dilation in poorly perfused vessels (and hence, $S_{\mathrm{m}}$ differs slightly from the original model [20, 25]).

In addition to being created, new vessels can also be removed by pruning. If a vessel is exposed to a WSS that is below a threshold $\left(\tau_{\mathrm{w}}{ }^{\text {crit }}\right)$ for a certain time ( $\left.T_{\text {prune }}\right)$, we remove that vessel from the system. In general, the vascular adaptation algorithm includes the following steps. First, the vessel radii evolve according to (3.21). Then we calculate the flows in the network. In contrast to the previous model [20], the evolution of the radius is considered separately and is not iterated until a steady state is reached.

\subsubsection{Computational Algorithm}

The basis of our model is a regular grid that subdivides the simulation domain into cellular automaton lattice sites. Each lattice site can be occupied by several biological cells and vessels. Figure 3.1 shows the high degree of coupling between the different spatial scales. We enumerate the main steps below:

1. Initialisation (vascular and cellular layers) We specify an initial vascular network as a system of straight pipes with fixed inflow and outflow nodes and prescribed pressures. We also prescribe the amount of haematocrit that enters through each inlet, and the initial location of the different cell types in the cellular automaton domain.

2. Update cells, oxygen and VEGF (diffusible, cellular and subcellular layers)

- Calculation of oxygen concentration (diffusible layer) The reactiondiffusion equation (3.14) is used to calculate the quasi-stationary oxygen concentration $c_{02}(t, \mathbf{x})$ in the simulation domain. Oxygen consumption by normal and cancer cells is included in (3.14) via sink terms, assuming firstorder kinetics. On the other hand, perfused blood vessels deliver oxygen to the tissue and thus account for oxygen sources. 
- Calculation of cell cycle, p53 and VEGF ODEs (subcellular layer) The subcellular layer is coupled to its local environment via the oxygen concentration. Oxygen drives the cell cycle of individual cells, whose current state is described by the time-dependent concentrations of the proteins Cdh1, cycCDK, p27, npRB and the cell mass $M$. Internal p53 and VEGF concentrations are also considered. All subcellular variables are modelled by the coupled systems of non-linear ODEs (3.1)-(3.7).

- Check for cell division (cellular layer) Cells divide if their Cdh1 and cycCDK concentrations are under, respectively, over a predefined threshold [see (3.10)].

- Cell movement (cellular layer) Vascular tip cells perform a biased random walk through the tissue. The probability of moving in a certain direction is influenced by the local VEGF gradient and cell densities [see (3.12)]. The motility of normal and cancer cells is also included via (3.12).

- Calculation of VEGF concentration (diffusible layer) Quiescent tumour cells and hypoxic normal cells produce VEGF, and so contribute to the source term in the reaction-diffusion equation (3.15) for the VEGF concentration $c_{\text {VEGF }}(t, \mathbf{x})$. VEGF is removed by the vascular system.

- Check for cell quiescence (cellular layer) Tumour cells enter or leave a quiescent state depending on the internal cell p27 concentration, which is described in (3.4). Oxygen is the external factor that influences the level of p27.

- Check for cell death (cellular layer) Normal cells die if their subcellular p53 concentration exceeds a threshold value. If a normal cell is surrounded by a high number of tumour cells, then its p53 threshold for cell death is reduced [see (3.8)]. This models the degradation of a tumour's environment by tumour cells. Tumour cells die if they are quiescent for a certain period of time; unlike normal cells, their death is not influenced by p53.

3. Update vasculature (cellular and vascular layer) The vascular system continually remodels and evolves in response to external and internal stimuli:

- Check for new tip cells (cellular layer) A raised VEGF concentration in the tissue stimulates the vasculature to form new sprouts. The probability that new sprouts emerge is specified by (3.11) and is an increasing function of the local extracellular VEGF concentration.

- Check for anastomosis (cellular layer) New vessels are formed if sprouts connect to other sprouts or to the pre-existing vascular network.

- Vessel pruning (vascular layer) Vessels that are underperfused $\left(\tau_{\mathrm{w}}<\tau_{\mathrm{w}}^{\text {crit }}\right.$ ) for a certain period of time ( $\left.T_{\text {prune }}\right)$ are removed from the vascular network.

- Calculation of radius adaptation (vascular layer) The vessel radii are updated at each timestep according to (3.21). The change in vessel radii is influenced by haemodynamic and metabolic stimuli as well as the general tendency of vessels to shrink [see (3.22)-(3.25)]. 
- Calculation of pressures and flows within vasculature (vascular layer) Poiseuille's flow (3.16) is considered in each branch of the vascular network, and the pressure at each node is calculated by applying conservation of mass. The haematocrit is assumed to split symmetrically at bifurcations.

In 1 , the successive cellular automaton model is initialized. Then 2 and 3 are carried out on each time interval $\Delta t$ until the final simulation time is reached.

\subsection{Simulations}

The results from a typical simulation, showing the development of a tumour and its associated network of blood vessels, are depicted in Fig. 3.2. Simulations were performed on a $50 \times 50 \times 50$ lattice with spacing $40 \mu \mathrm{m}$, which corresponds to a $2 \mathrm{~mm} \times 2 \mathrm{~mm} \times 2 \mathrm{~mm}$ cube of tissue. For the following simulations, each lattice site can be occupied by at most one cell (either normal or cancerous), which implies that, for the grid size used $(40 \mu \mathrm{m})$, the tissue is not densely packed. A small tumour was implanted at $t=0 \mathrm{~h}$ in a population of normal cells perfused by two parallel parent vessels with countercurrent flow (i.e. the pressure drops and hence flows are in opposite directions). Initially, insufficient nutrient supply in regions at distance from the vessels causes widespread death of the normal cells. The surviving tumour cells reduce the p53 threshold for death of normal cells, which further increases the death rate of the normal cells and enables the tumour to spread. Initially, due to inadequate vascularisation, most of the tumour cells are quiescent and secrete VEGF which stimulates an angiogenic response. After a certain period of time, the quiescent cells die and only a small vascularised tumour remains, encircling the upper vessel. The tumour expands preferentially along this vessel, in the direction of highest nutrient supply. Diffusion of VEGF throughout the domain stimulates the formation of new capillary sprouts from the lower parent vessel. When the sprouts anastomose with other sprouts or existing vessels, the oxygen supply increases, enabling the normal cell population to recover. Because the tumour cells consume more oxygen than normal cells and they more readily secrete VEGF under hypoxia, VEGF levels are higher inside the tumour, and the vascular density there is much higher than in the healthy tissue. The tumour remains localised around the upper vessel until new vessels connect the upper and lower vascular networks. Thereafter, the tumour cells can spread to the lower region of the domain until eventually the domain is wholly occupied by cancer cells and their associated vasculature.

As a further step, we document preliminary results of a vascular tumour growth simulation for which the initial vascular geometry was taken from multiphoton fluorescence microscopy (a detailed description of the experimental setting can be found in [22]). The aim here is to integrate the mathematical model with in vivo 
a
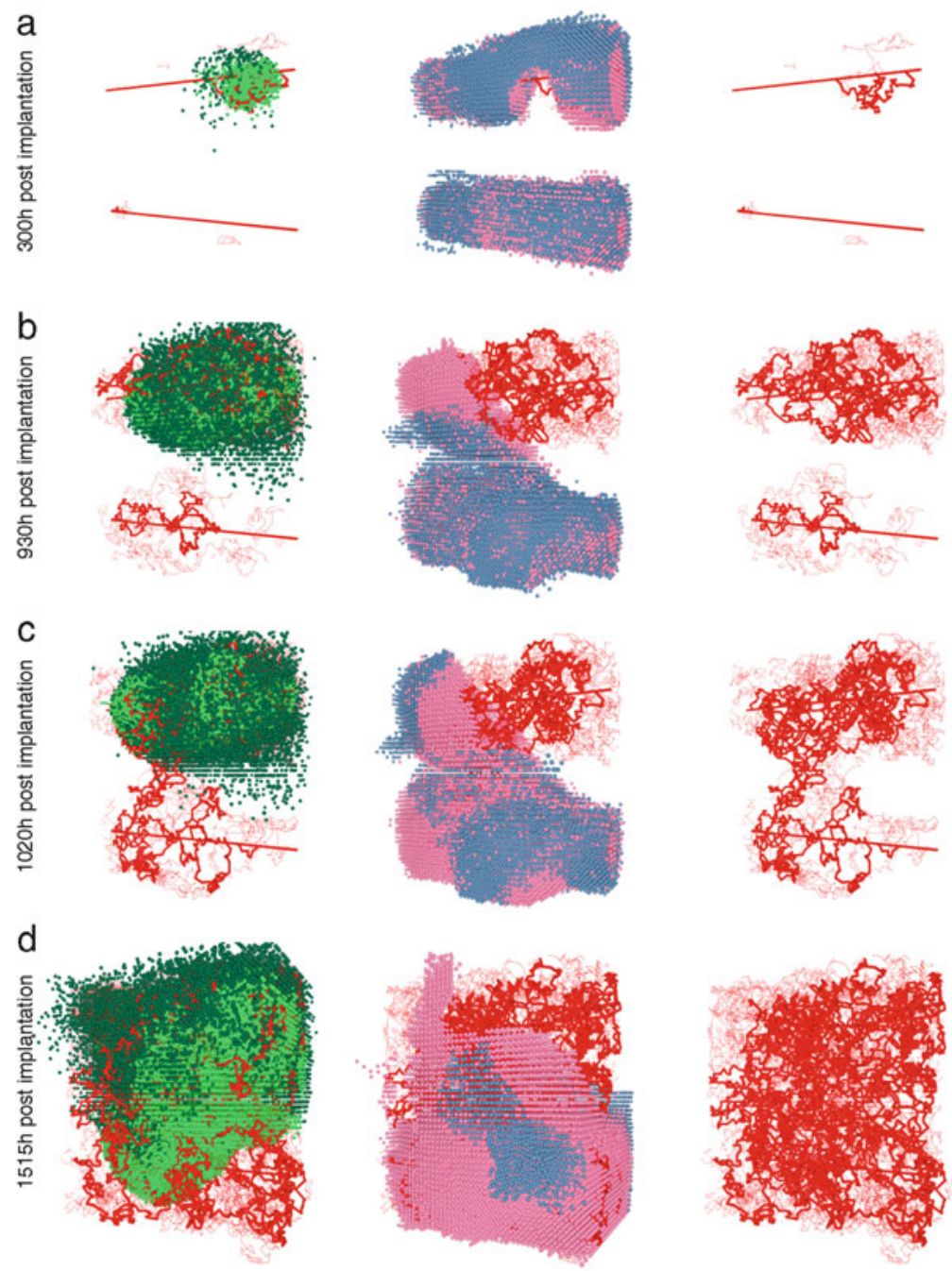

\section{- Tumour cells \\ - Quiescent tumour cells Vessels}

- Normal cells

- Hypoxic normal cells

Fig. 3.2 Tumour growth in healthy tissue. The tumour cells and vasculature are depicted in the left column, the vasculature and normal cells in the middle column and the vessel network in the right column. The figure shows a realisation of a $50 \times 50 \times 50$ domain with a cube of tumour cells implanted in healthy tissue initially with two straight vessels 


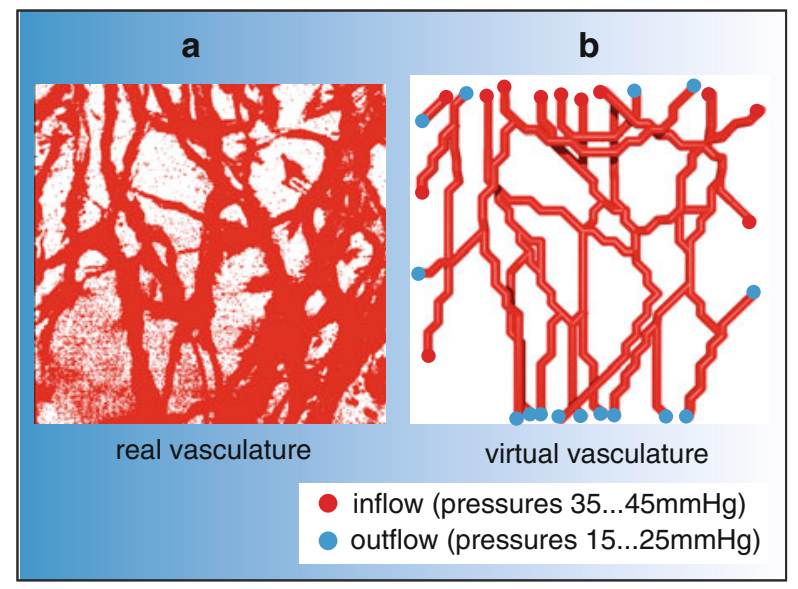

Fig. 3.3 Image reconstruction. We reconstructed the vascular network by applying the following strategy. 3D multiphoton fluorescence microscopy images (a) taken from mouse models in vivo formed the basis of our geometrical reconstruction. Based on the data, we reconstructed the vascular graph model that describes the connectivity of the vascular network. (b ) We assigned inflow (red points) and outflow (blue points ) nodes at various pressures in order to obtain a persistent and stable network. The vascular graph is characterised by the spatial coordinates of the nodes and the connections between them

experimental data. Experimental data defining a vascular network associated with a tumour were obtained by implanting a tumour construct comprising a central core of human breast cancer cells surrounded by rat microvessel fragments, embedded in a collagen matrix into a mouse dorsal window chamber. The cancer cells and rat microvascular cells express different fluorescent proteins so that, following implantation, the tumour and its vascular network can be visualised.

We used experimental data to reconstruct the vascular graph model, locating nodes in the vessel centres and connecting them by edges. We embed the vascular system into healthy tissue and then simulate vessel adaptation until a steady state is reached. This example provides proof of concept.

Currently in the computational models, the vasculature is embedded in a healthy tissue into which a small tumour is implanted and its evolution is studied. A projection of a 3D image set of the tissue is presented on the left-hand side of Fig. 3.3, while the virtual reconstruction is shown on the right-hand side. In Fig. 3.4, we observe that the tumour expands radially into the surrounding healthy tissue which it degrades by decreasing the p53 death threshold for normal cells. Normal cells in the lower left and right corners of the simulation domain (first column) are exposed to low oxygen (hypoxia), and hence produce VEGF which induces an angiogenic response in our model. While the new vessel in the lower left corner is persistent and increases in radius, the vessel in the lower right corner is pruned back. In this case, pruning occurs because the new blood vessel connects vessels from the initial network that have similar pressures. In general, the normal cells are adequately nourished by oxygen as only a few hypoxic cells can be 

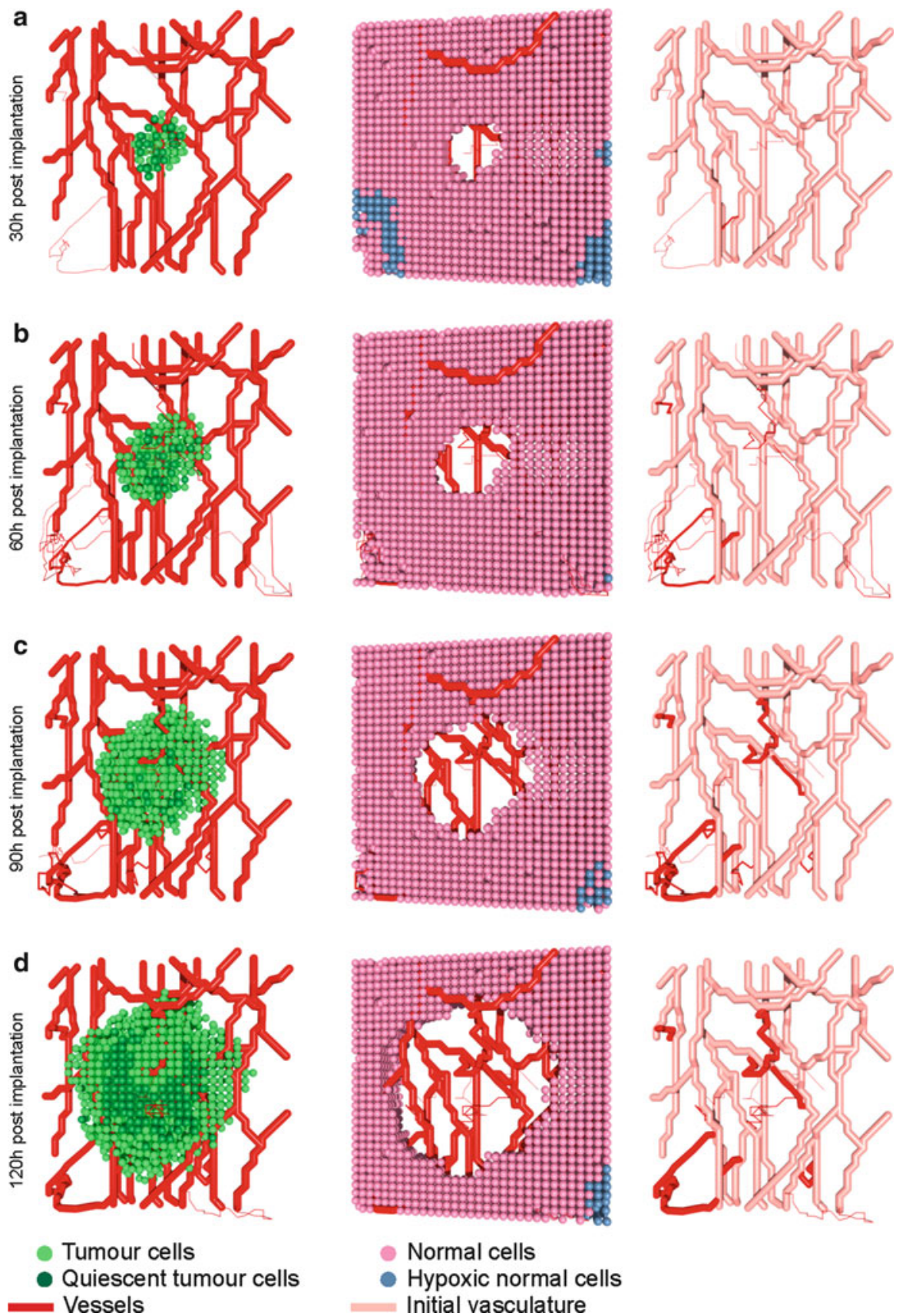

Initial vasculature

Fig. 3.4 Proof of concept: tumour growth in an experimentally derived vascular network. (a -d ) show the computed temporal evolution of a tumour in a real vascular network embedded in normal tissue. As initial condition, we have taken a vascular network from multiphoton fluorescence 
observed in simulations with normal cells only. In contrast, we find a high percentage of quiescent cancer cells in all states of tumour growth, leading to further angiogenesis in our simulations (see Fig. 3.4). The dark red vessels in row 3 indicate new vessels that develop after tumour implantation. In conclusion, our model with the chosen parameter values predicts an increase in the vascular density following tumour implantation.

\subsection{Conclusions}

In this chapter we have presented a multiscale model of vascular tumour growth and angiogenesis. After the introduction, the mathematical model was presented in Sect. 3.2 where we gave a detailed description of the mathematical models on the different length scales. Finally, we introduced the computational algorithm that we use to simulate the model. In the third section, simulation results were shown. We started by considering the growth of a tumour nested in healthy tissue initially perfused by two straight and parallel vessels and then studied the evolution of cells and the vascular system. As proof of concept, we then used an experimentally derived vessel network to initialise a simulation of tumour growth and angiogenesis. To the best of our knowledge, this is the first time this has been done-Secomb, Pries and co-workers (e.g. [31, 26]) have used such networks to study structural adaptation alone. Our work paves the way for further research which will be more closely linked with experimental data. In particular, it would be of great interest to compare our model simulations with experimental data from two or more time points. The first time point defining the initial conditions for the simulations and data from later time points used to test the model's predictive power or to estimate parameter values. We would not expect to obtain a detailed match at later time points, since we simulate a stochastic system, but we would expect agreement between experimental and simulated values for certain characteristics, such as vessel volume fractions and the distributions of vessel radii and segment lengths.

One problem is the large number of parameters contained in multiscale models such as ours. This makes it nontrivial to parametrise them. One strategy would be to start by parametrising small and well-defined submodels independently of each other. In this way, it should then be possible to determine whether coupling the submodels together gives physiologically realistic results or if additional effects have to be incorporated. Another important issue is determining the influence that each system parameter has on the simulation results. This could be established by performing a comprehensive parameter sensitivity analysis. Such knowledge would

Fig. 3.4 (continued) microscopy and embedded it in a $32 \times 32 \times 6$ cellular automaton domain. In the first column, the tumour expands radially, and degrades the healthy tissue (second column). The predicted adaptations of the vascular system are shown in the third column where the experimentally derived network is shown in light red, while the new vessels are coloured in red 
enable us to identify those biophysical mechanisms that dominate the system dynamics and to use this information to derive simpler models which exhibit the same behaviour. Unfortunately, simulations are very time-consuming - the simulation shown in Fig. 3.2 takes several days on a desktop computer, and then several realisations of the Monte Carlo simulation have to be carried out for a statistical analysis. Therefore, future optimisations and the parallelisation of the computer programme are essential. One also has to investigate to which extent the models are overdetermined, meaning that changes in different parameters lead to the same pattern in the simulations.

Beside these limitations, multiscale models build promising frameworks for future developments. They enable us to investigate how processes operating on different space and time scales interact and to study the effect that such interactions have on the overall system dynamics. They also enable researchers in different areas to link and couple their models. To simplify this model exchange, model interfaces have to be defined and standardised. Equally, multiscale models can be used to develop and parametrise simpler continuum models that can be solved more efficiently. Most current multiscale models generate qualitatively accurate and meaningful results, and, therefore, they can be applied to identify sensitive mechanisms that then stimulate biological experiments.

Acknowledgements $\mathrm{HMB}$, MRO and HP acknowledge financial support by the Marie Curie Network MMBNOTT (Project No. MEST-CT-2005-020723). RAG and PKM acknowledge partial support from NIH/NCI grant U54CA143970. HP, AL and MR thank the BMBF-Funding Initiative FORSYS Partner: "Predictive Cancer Therapy". In vivo window chamber work was funded in part by Moffitt Cancer Center PS-OC NIH/NCI U54CA143970. This publication was based on work supported in part by Award No. KUK-C1-1013-04, made by King Abdullah University of Science and Technology (KAUST).

\section{References}

1. Alarcón T, Byrne HM, Maini PK (2003) A cellular automaton model for tumour growth in an inhomogeneous environment. J Theor Biol 225:257-274

2. Alarcón T, Byrne HM, Maini PK (2004) A mathematical model of the effects of hypoxia on the cell-cycle of normal and cancer cells. J Theor Biol 229(3):395-411

3. Alarcón T, Byrne HM, Maini PK (2005) A multiple scale model for tumor growth. Multiscale Model Sim 3:440-475

4. Alarcón T, Owen MR, Byrne HM, Maini PK (2006) Multiscale modelling of tumour growth and therapy: The influence of vessel normalisation on chemotherapy. Comput Math Method M7(2-3):85-119

5. Anderson ARA (2005) A hybrid mathematical model of solid tumour invasion: The importance of cell adhesion. Math Med Biol 22:163-186

6. Anderson ARA, Chaplain MAJ (1998) Continuous and discrete mathematical models of tumor-induced angiogenesis. Bull Math Biol 60(5):857-899

7. Arakelyan L, Vainstein V, Agur Z (2002) A computer algorithm describing the process of vessel formation and maturation, and its use for predicting the effects of anti-angiogenic and anti-maturation therapy on vascular tumor growth. Angiogenesis 5(3):203-14 
8. Arakelyan L, Merbl Y, Agur Z (2005) Vessel maturation effects on tumour growth: validation of a computer model in implanted human ovarian carcinoma spheroids. Eur J Cancer 41 (1):159-167

9. Breward CJW, Byrne HM, Lewis CE (2003) A multiphase model describing vascular tumour growth. Bull Math Biol 65:609-640

10. Clark ER (1918) Studies on the growth of blood-vessels in the tail of the frog larva - by observation and experiment on the living animal. Am J Anat 23(1):37-88

11. Drasdo D, Jagiella N, Ramis-Conde I, Vignon-Clementel I, Weens W (2010) Modeling steps from a benign tumor to an invasive cancer: Examples of intrinsically multi-scale problems. In: Chauviere A, Preziosi L, Verdier C (eds) Cell mechanics: From single scale-based models to multiscale modeling. Chapman \& Hall/CRC, pp 379-417

12. Folkman J (1971) Tumour angiogenesis - therapeutic implications. New Engl J Med 285:1182-1186

13. Folkman J, Klagsburn M (1987) Angiogenic factors. Science 235:442-447

14. Frieboes HB, Lowengrub JS, Wise S, Zheng X, Macklin P, Bearer E, Cristini V (2007) Computer simulation of glioma growth and morphology. Neuroimage 37(S1):59-70

15. Lee DS, Rieger H, Bartha K (2006) Flow correlated percolation during vascular remodeling in growing tumors. Phys Rev Lett 96(5):058,104-4

16. Lloyd BA, Szczerba D, Rudin M, Székely G (2008) A computational framework for modelling solid tumour growth. Phil Trans R Soc A 366:3301-3318

17. Macklin P, McDougall S, Anderson AR, Chaplain MAJ, Cristini V, Lowengrub J (2009) Multiscale modelling and nonlinear simulation of vascular tumour growth. J Math Biol 58:765-798

18. Mantzaris N, Webb SD, Othmer HG (2004) Mathematical modelling of tumour angiogenesis: A review. J Math Biol 49:111-187

19. McDougall SR, Anderson ARA, Chaplain MAJ (2006) Mathematical modelling of dynamic adaptive tumour-induced angiogenesis: Clinical implications and therapeutic targeting strategies. J Theor Biol 241(3):564-89

20. Owen MR, Alarcón T, Maini PK, Byrne HM (2009) Angiogenesis and vascular remodelling in normal and cancerous tissues. J Math Biol 58:689-721

21. Owen M, Stamper I, Muthana M, Richardson G, Dobson J, Lewis C, Byrne H (2011) Mathematical modeling predicts synergistic antitumor effects of combining a macrophagebased, hypoxia-targeted gene therapy with chemotherapy. Cancer Res 71(8):2826

22. Perfahl H, Byrne H, Chen T, Estrella V, Alarcón T, Lapin A, Gatenby R, Gillies R, Lloyd M, Maini P, et al (2011) Multiscale modelling of vascular tumour growth in 3d: The roles of domain size and boundary conditions. PloS one 6(4):e14,790. doi:10.1371/journal. pone. 0014790

23. Plank MJ, Sleeman BD (2003) A reinforced random walk model of tumour angiogenesis and anti-angiogenic strategies. Math Med Biol 20(2):135-181

24. Plank M, Sleeman B (2004) Lattice and non-lattice models of tumour angiogenesis. Bull Math Biol 66(6): 1785-1819

25. Pries AR, Secomb TW, Gaehtgens P (1998) Structural adaptation and stability of microvascular networks: Theory and simulations. Am J Physiol 275(2 Pt 2):H349-H360

26. Pries A, Reglin B, Secomb T (2001) Structural adaptation of microvascular networks: Functional roles of adaptive responses. Am J Physiol 281:H1015-H1025

27. Pugh C, Rattcliffe P (2003) Regulation of angiogenesis by hypoxia: Role of the hif system. Nature Med 9:677-684

28. Resnick N, Yahav H, Shay-Salit A, Shushy M, Schubert S, Zilberman L, Wofovitz E (2003) Fluid shear stress and the vascular endothelium: For better and for worse. Progr Biophys Mol Biol 81:177-199

29. Risau W (1997) Mechanisms of angiogenesis. Nature 386:871-875

30. Schaller G, Meyer-Hermann M (2005) Multicellular tumor spheroid in an off-lattice VoronoiDelaunay cell model. Phys Rev E 71:1-16 
31. Secomb TW, Hsu R, Beamer NB, Coull BM (2000) Theoretical simulation of oxygen transport to brain by networks of microvessels: Effects of oxygen supply and demand on tissue hypoxia. Microcirculation 7:237-247

32. Shirinifard A, Gens JS, Zaitlen BL, Poplawski NJ, Swat M (2009) 3D Multi-Cell simulation of tumor growth and angiogenesis. PLoS ONE 4(10):e7190. doi:10.1371/journal.pone.0007190

33. Stéphanou A, McDougall SR, Anderson ARA, Chaplain MAJ (2005) Mathematical modelling of flow in $2 \mathrm{~d}$ and $3 \mathrm{~d}$ vascular networks: Applications to anti-angiogenic and chemotherapeutic drug strategies. Math Comp Modelling 41(10):1137-1156

34. Stokes CL, Lauffenburger DA (1991) Analysis of the roles of microvessel endothelial cell random motility and chemotaxis in angiogenesis. J Theor Biol 152(3):377-403

35. Tracqui P (2009) Biophysical models of tumour growth. Rep Prog Phys 72(5):056,701

36. Tyson JJ, Novak B (2001) Regulation of the eukaryotic cell cycle: Molecular antagonism, hysteresis, and irreversible transitions. J Theor Biol 210:249-263

37. Zheng X, Wise SM, Cristini V (2005) Nonlinear simulation of tumor necrosis, neovascularization and tissue invasion via an adaptive finite-element/level set method. Bull Math Biol 67(2):211-259 